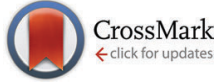

Cite this: Mol. BioSyst., 2015, 11, 1454

Received 3rd February 2015, Accepted 30th March 2015

DOI: 10.1039/c5mb00102a

www.rsc.org/molecularbiosystems

\title{
Probing the effect of minor groove interactions on the catalytic efficiency of DNAzymes 8-17 and $10-23 \dagger$
}

\author{
Michael H. Räz and Marcel Hollenstein*
}

\begin{abstract}
DNAzymes (Dz) 8-17 and 10-23 are two widely studied and well-characterized RNA-cleaving DNA catalysts. In an effort to further improve the understanding of the fragile interactions and dynamics of the enzymatic mechanism, this study examines the catalytic efficiency of minimally modified DNAzymes. Five single mutants of Dz8-17 and Dz10-23 were prepared by replacing the adenine residues in the corresponding catalytic cores with 3-deazaadenine units. Kinetic assays were used to assess the effect on the catalytic activity and thereby identify the importance of hydrogen bonding that arises from the N3 atoms. The results suggest that modifications at $A_{15}$ and $A_{15.0}$ of Dz8-17 have a significant influence and show a reduction in catalytic activity. Modification at each location in Dz10-23 results in a decrease of the observed rate constants, with $\mathrm{A}_{12}$ appearing to be the most affected with a reduction of $\sim 80 \%$ of $k_{\text {obs }}$ and $\sim 25 \%$ of the maximal cleavage rate compared to the wild-type DNAzyme. On the other hand, modification of $\mathrm{A}_{12}$ in Dz8-17 showed an $\sim 130 \%$ increase in $k_{\text {obs }}$, thus unraveling a new potential site for the introduction of chemical modifications. A pH-profile analysis showed that the chemical cleavage step is rate-determining, regardless of the presence and/or location of the mutation. These findings point towards the importance of the N3-nitrogens of certain adenine nucleotides located within the catalytic cores of the DNAzymes for efficient catalytic activity and further suggest that they might directly partake in maintaining the appropriate tertiary structure. Therefore, it appears that minor groove interactions constitute an important feature of DNAzymes as well as ribozymes.
\end{abstract}

\section{Introduction}

DNAzymes (Dz or DNA enzymes) are single stranded DNA molecules, which are able to catalyze chemical reactions. ${ }^{1-5}$ Since the first discovery of these catalytic DNA molecules by Gerald Joyce and Ronald Breaker in 1994 by application of in vitro selection techniques, ${ }^{6}$ a broad range of DNAzymes was discovered, catalyzing a variety of reactions including $\mathrm{RNA}^{7,8}$ and DNA-cleavage, ${ }^{9,10}$ DNA-mediated ester bond cleavage, ${ }^{11}$ phosphomonoester hydrolysis of amino acid side chains, ${ }^{12} \mathrm{C}-\mathrm{C}$ bond formation, ${ }^{13}$ generation of nucleopeptide linkages ${ }^{14}$ formation of linear, branched or lariat RNA, ${ }^{15,16}$ DNA phosphorylation ${ }^{17}$ and labelling, ${ }^{18}$ and thymine dimer photoreversion. ${ }^{19}$ Thus, DNA molecules can be coerced into catalyzing a broad array of reactions that involve not only oligonucleotide-based substrates where Watson-Crick base pairing dictates the interaction of the enzyme and the substrate, but also non-nucleotidic substrates.

Department of Chemistry and Biochemistry, University of Bern, Freiestrasse 3, 3012 Bern, Switzerland. E-mail: hollenstein@dcb.unibe.ch

$\dagger$ Electronic supplementary information (ESI) available: UV melting experiments, kinetic assays, and pH-rate profiles. See DOI: 10.1039/c5mb00102a
Moreover, the inherent folding properties of DNA, its intrinsic capacity for replication, along with the cheap and efficient production of DNA oligonucleotides make DNAzymes alluring targets for potential applications in numerous fields including chemical biology, biotechnology, and medicinal chemistry. ${ }^{20,21}$ However, despite the advantages of generating DNAzymes via in vitro experiments, there are also major drawbacks to these catalytic entities, especially when considering in vivo treatments and medical applications. Indeed, like any other oligonucleotides, DNAzymes are susceptible to degradation by nucleases. Moreover, efficient catalysis $\left(k_{\mathrm{cat}} / K_{\mathrm{M}}\right.$ values $\left.\geq 10^{8} \mathrm{~min}^{-1} \mathrm{M}^{-1}\right)$ usually relies on the presence of high concentrations of metal cofactors $\left(\mathrm{M}^{2+}\right)$ exceeding the levels present in a cell. ${ }^{22}$

In order to alleviate these drawbacks, chemical modifications can be incorporated into the scaffold of DNAzymes. Efforts towards this goal usually proceed either by (1) including modified nucleoside triphosphates ( $\left.\mathrm{dN}^{*} \mathrm{TPs}\right)$ in combinatorial selection experiments $^{23-32}$ or by (2) incorporating nucleoside analogs into the catalytic core or binding arms of selected unmodified DNAzymes via solid-phase oligonucleotide synthesis. ${ }^{33-41}$ The advantage of solid-phase synthesis is that the chemical nature (and thus the purpose of modification), the location, and the exact number 


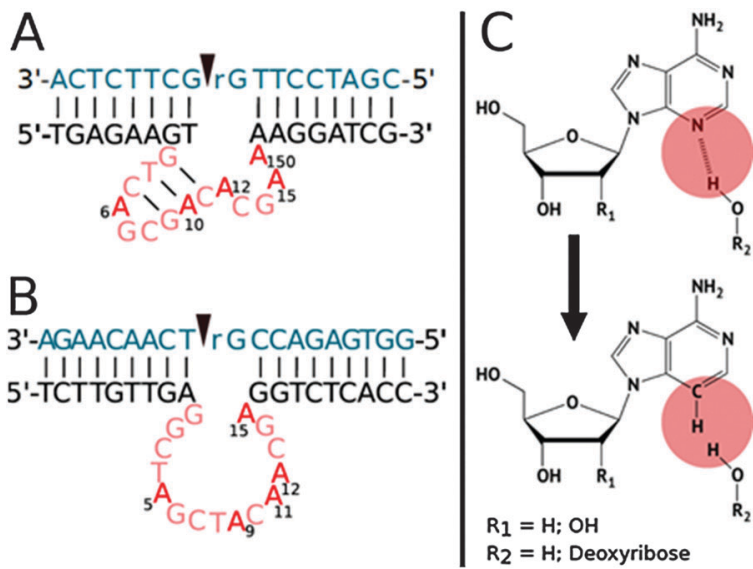

Fig. 1 Hypothetical secondary structures of Dz8-17 (A) and Dz10-23 (B). Both have a $15 \mathrm{nt}$ catalytic core (shown in red) and two substrate binding arms (black). The substrate (blue) is bound via Watson-Crick base-pairing and cleavage occurs at the scissile ribophosphodiester linkage (arrow) (C) A carbon atom is incorporated at the 3-position of the purine ring, preventing the formation of hydrogen bonds.

of analogs can be easily modulated. However, since all combinatorial evolution experiments including SELEX follow the principle "you get what you select for", ${ }^{42}$ DNAzymes display finely tuned catalytic surfaces where even minute alterations can lead to a loss of activity. ${ }^{27}$ Consequently, incorporation of modified nucleotides into the scaffold of DNAzymes is a difficult undertaking since an investigation needs to be done to assess the effect that the chemical functionalities might have on the catalytic efficiency. Therefore, investigations on the mechanism and the dynamics of DNAzymes and their catalytic core, in particular, are of great interest. ${ }^{43-45}$

In this context, Dz8-17 and 10-23 are two well-known and widely used RNA-cleaving DNAzymes each consisting of a catalytic core with 15 nucleotides and two substrate binding arms (Fig. 1). ${ }^{7}$

Dz8-17 possesses a stem-loop motif in the catalytic core, and mutagenesis experiments revealed that certain nucleotides such as $A_{6}, G_{7}$, and $G_{14}$ were strictly conserved and could not be altered or substituted, while other residues, such as $A_{12}$ and $A_{15}$, could be varied without causing a severe depletion in catalytic efficiency. ${ }^{8,46,47}$ Similarly, even though Dz10-23 seems to be devoid of structural motifs, various residues of the catalytic core have been shown to be highly conserved (such as $G_{1}, A_{5}$, and $G_{6}$ ), while other locations ( $T_{8}$ and $A_{9}$, particularly) are thought to be tolerant to rather important modifications. ${ }^{38,47-49}$ Recently, Li et al. refined the understanding of the critical positions by incorporating an abasic site and a C3 spacer within the catalytic cores of both DNAzymes. ${ }^{47}$

In the present study, the goal was to hone the understanding of the fragile interactions and dynamics of the enzymatic mechanism by focusing on other interactions involving nucleotides. Indeed, next to the well-known Watson-Crick and Hoogsteen base pairings, the N3-nitrogen in the purine ring of a guanine, and even more importantly in adenine, is involved in the formation of the so-called A-minor interactions (adenine minor groove interactions).
These interactions were first discovered in the secondary structures of rRNAs $^{50}$ and it could be shown that the A-minor motif in RNA is very common and mostly consists of an unpaired adenine inserted into the minor groove of RNA helices with a high $\mathrm{C}-\mathrm{G}$ content. ${ }^{51-53}$ A-minor motifs help to stabilize interactions between RNA duplexes, contacts of loops and helices or other conformations since the N3-nitrogen can act as a hydrogen bond acceptor that can facilitate interactions with both the 2 '-OH groups and exocyclic amines of guanine or cytosine nucleotides. ${ }^{52}$ Studies carried out by McLaughlin et al. focusing on the N3-nitrogens of conserved adenine nucleotides in the hammerhead ribozyme, a naturally occurring ribozyme catalyzing the self-cleavage of RNA substrates, showed that A-minor interactions play an important role in ribozymes and are crucial for maintaining the enzymatic function. ${ }^{54}$ Even though no A-minor motifs have formally been identified in DNA so far, adenine N3-nitrogen atoms have been shown to play a crucial role in the hydration pattern of the minor groove of DNA (by hydrogen bonding to the primary water layer) and thus in the overall structure and stability of DNA duplexes. ${ }^{55,56}$ Herein, we systematically replaced all the adenine units of the catalytic cores of the 8-17 and 10-23 DNAzymes with 2'-deoxy-3deazaadenosine units, an analog that is devoid of a site that allows for hydrogen-bonding in the minor groove. Such a substitution caused a reduction of the observed rate constants when introduced at specific locations of both DNAzymes, suggesting that minor groove interactions play an active role in the mechanism of DNA-based catalysts.

\section{Results and discussion}

\section{Nucleotide substitution in the catalytic core and thermal denaturation experiments}

In order to investigate on the effect of minor groove interactions on the catalytic efficiency of DNAzymes, the phosphoramidite of 2'-deoxy-3-deazaadenosine was synthesized according to a known synthetic pathway. ${ }^{56,57}$ This nucleoside analog was then incorporated into the catalytic core of the 8-17 and 10-23 DNAzymes via a standard automated solid-phase oligonucleotide synthesis in lieu of each of the natural adenine residues (see ESI $\dagger$ ). Since numerous variants of both the 8-17 and 10-23 DNAzymes exist, all obtained by selection under different conditions or by reselection from enriched libraries, ${ }^{1,58,59}$ we focused on the species used by Li et al. for the sake of comparison (Fig. 1). ${ }^{47}$ Thus, the wildtype Dz8-17 used in this study consists of a 15-nucleotide catalytic core and two substrate binding arms. The catalytic domain has a stem formed by three Watson-Crick base-pairs, a loop containing an AGC triplet and 5 unpaired nucleotides to complete the catalytic core (Fig. 1A), for a total of 5 adenine nucleotides (i.e. $\mathrm{A}_{6}, \mathrm{~A}_{10}, \mathrm{~A}_{12}, \mathrm{~A}_{15}$, and $\left.\mathrm{A}_{15.0}\right)$. Similarly, the wild-type Dz10-23 also has a 15-nucleotide catalytic core but built up of only unpaired nucleotides and no base-pairing motifs for a total of 5 adenine units (i.e. $\mathrm{A}_{5}, \mathrm{~A}_{9}, \mathrm{~A}_{11}, \mathrm{~A}_{12}$, and $\mathrm{A}_{15}$; Fig. $1 \mathrm{~B}$ ). The resulting mutants were named 817_X and 1023_X, where X refers to the position of the substituent within the catalytic unit (Fig. 1). 
Table $1 T_{m}$ data for the natural and modified duplex and single strands of Dz8-17

\begin{tabular}{lllllc}
\hline & \multicolumn{2}{l}{ Duplex $^{a}$} & & \multicolumn{2}{c}{ Single strand } \\
\cline { 2 - 3 } Dz8-17 & Avg. $T_{\mathrm{m}}\left({ }^{\circ} \mathrm{C}\right)$ & $\Delta T_{\mathrm{m}}\left({ }^{\circ} \mathrm{C}\right)$ & & Avg. $T_{\mathrm{m}}\left({ }^{\circ} \mathrm{C}\right)$ & $\Delta T_{\mathrm{m}}\left({ }^{\circ} \mathrm{C}\right)$ \\
\hline 817_WT & 64.3 & 0.0 & & 48.7 & 0.0 \\
817_6 & 63.3 & -1.0 & 52.2 & 3.5 \\
817_10 & 63.3 & -1.0 & & 51.7 & 3.0 \\
817_12 & 63.3 & -1.0 & & 45.7 & -3.0 \\
817_15 & 63.2 & -1.0 & & 57.2 & 8.5 \\
817_150 & 64.0 & -0.4 & & 54.5 & 6.8
\end{tabular}

${ }^{a}$ Complement: 5'-CGATCCTTTTCGTGTCGCTGACACTTCTCA-3' .

In order to assess the effect of the substitution on the overall stabilization of duplexes, thermal denaturation experiments of natural and modified oligonucleotides were carried out (Tables 1, 2 and ESI $\dagger$ ). To better estimate the effect of deletion of the N3 atom, the sequences of Dz8-17 and 10-23 were paired with the corresponding complementary sequence ${ }^{60}$ and the obtained $T_{\mathrm{m}}$ values are summarized in Table 1 for Dz8-17 and in Table 2 for Dz10-23. We decided to measure the melting temperatures of modified oligonucleotides corresponding to both DNAzymes paired with the complementary strand instead of a noncleavable substrate since the modifications are all located within the catalytic core. The inclusion of the modification had only a marginally destabilizing effect on the duplex stability $\left(\Delta T_{\mathrm{m}} \sim-1.0{ }^{\circ} \mathrm{C} /\right.$ modification $)$ in the case of Dz8-17, which compares favorably to other reports where stronger destabilization of duplexes was observed. ${ }^{55,56,61}$ Surprisingly, the melting curves of the single strands also showed cooperative and reversible melting events (Table 1 and Fig. S2, ESI $\dagger$ ). Indeed, $T_{\mathrm{m}}$ values at around $\sim 50{ }^{\circ} \mathrm{C}$ are consistently observed, with the mutant 817_15 displaying the highest $T_{\mathrm{m}}$ value $\left(57.2{ }^{\circ} \mathrm{C}\right)$ while the lowest $T_{\mathrm{m}}$ value was observed for 817_12 $\left(T_{\mathrm{m}}=45.7{ }^{\circ} \mathrm{C}\right)$. These rather high $T_{\mathrm{m}}$ values might be ascribed to the destacking of the secondary structures, most likely the thermal denaturation of the putative $3 \mathrm{bp}$ stem of the central triloop. ${ }^{8}$ Indeed, very short hairpin systems consisting of only two G-C base pairs and a shear G-A base pair have been shown to form extraordinarily stable systems $\left(T_{\mathrm{m}}>70{ }^{\circ} \mathrm{C}\right),{ }^{62,63}$ and therefore, the formation of the GTCAGCGAC stem-loop could account for the observed melting event.

On the other hand, the variations in $T_{\mathrm{m}}$ values obtained in the case of Dz10-23 are more marked than in the case of Dz8-17.

Table $2 T_{\mathrm{m}}$ data for the natural and modified duplex and single strands of Dz10-23

\begin{tabular}{llllll}
\hline & \multicolumn{2}{l}{ Duplex $^{a}$} & & \multicolumn{2}{c}{ Single strand } \\
\cline { 2 - 3 } Dz10-23 & Avg. $T_{\mathrm{m}}\left({ }^{\circ} \mathrm{C}\right)$ & $\Delta T_{\mathrm{m}}\left({ }^{\circ} \mathrm{C}\right)$ & & Avg. $T_{\mathrm{m}}\left({ }^{\circ} \mathrm{C}\right)$ & $\Delta T_{\mathrm{m}}\left({ }^{\circ} \mathrm{C}\right)$ \\
\hline 1023_WT & 65.0 & 0.0 & 37.0 & 0.0 \\
1023_5 & 63.0 & -2.0 & & 41.2 & 4.2 \\
1023_9 & 62.0 & -3.0 & & 39.7 & 2.7 \\
1023_11 & 62.3 & -2.8 & & 32.0 & -5.0 \\
1023_12 & 58.8 & -6.2 & & 35.7 & -1.2 \\
1023_15 & 60.7 & -4.4 & 35.3 & -1.7
\end{tabular}

${ }^{a}$ Complement: 5'-CGATCCTTTTCGTGTCGCTGACACTTCTCA-3'.
Indeed, the modified strands cause a destabilization of $2{ }^{\circ} \mathrm{C}$ to $6{ }^{\circ} \mathrm{C}$ as compared to the unmodified system (Table 2) and is in par with what has been observed in different sequence contexts. ${ }^{55,56}$ Thus, the introduction of single modifications in the Dz10-23 construct leads to a more pronounced effect than similar alterations brought to the core of Dz8-17. Most melting curves for Dz10-23 clearly show a second transition at around $40{ }^{\circ} \mathrm{C}$, which might be due to a change in the tertiary structure of the duplex and/or the single strand. As observed in the case of Dz8-17, the melting curves of the single strands of Dz10-23 show cooperative and reversible melting events, albeit with $T_{\mathrm{m}}$ values $\sim 10{ }^{\circ} \mathrm{C}$ lower than those observed for the Dz8-17 single strands. 1023_5 shows the most stable secondary self-structure $\left(T_{\mathrm{m}}=41.0{ }^{\circ} \mathrm{C}\right)$, while $1023 \_11$ displays the least stable selfstructure $\left(T_{\mathrm{m}}=32.0{ }^{\circ} \mathrm{C}\right)$. The second transition in the melting curves might be ascribed to the melting of a duplex formed by two single strands maintained together by the 6nt long palindromic sequence located within the catalytic core rather than any putative secondary structure as in the case of Dz8-17. ${ }^{64}$

\section{Effects of substitution on the catalytic activity}

In order to assess the effect of potential minor groove interactions on the catalytic activity, cleavage reactions were performed under single turnover conditions using a chimeric DNA/RNA substrate ${ }^{32} \mathrm{P}$-radiolabeled with both the wild-type and modified constructs. The results of the kinetic assays are shown in Fig. 2A for Dz8-17 and in Fig. 2B for Dz10-23. In addition, the values of the observed rate constants $\left(k_{\mathrm{obs}}\right)$ and maximal cleavage rates $\left(Y_{\mathrm{m}}\right)$ were derived from curve-fitting of the data and the results are summarized in Table 3 . The $k_{\mathrm{obs}}$ and $Y_{\mathrm{m}}$ values of all the modified constructs are normalized against those of the native DNAzymes.

For Dz8-17, it can be noted that the maximal cleavage yield for all constructs remains unchanged $\left(Y_{\mathrm{m}}=\sim 80 \%\right)$. The values of $k_{\text {obs }}$, on the other hand, vary substantially and depletions as well as improvements in the catalytic activity can be observed. Indeed, the catalytic efficiencies of three of the mutant
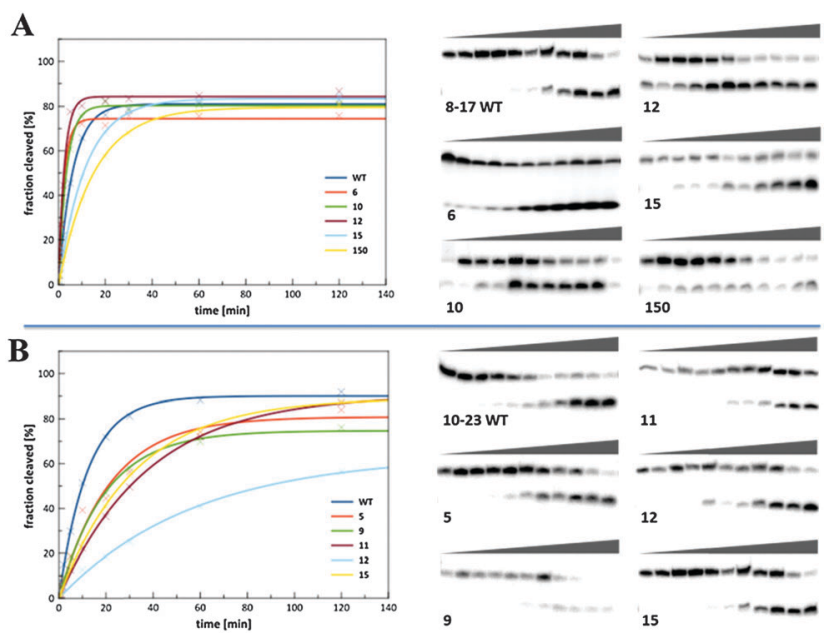

Fig. 2 Kinetic plots and PAGE gels of the kinetic assays of the wild type and modified constructs. (A) Dz8-17. (B) Dz10-23. 
Table $3 \quad k_{\text {obs }}$ and $Y_{m}$ values for the wild type and modified constructs of Dz8-17 and Dz10-23

\begin{tabular}{lllll}
\hline & $k_{\text {obs }}\left(\mathrm{min}^{-1}\right)$ & $Y_{\mathrm{m}}$ & Rel. $k_{\text {obs }}$ & Rel. $Y_{\mathrm{m}}$ \\
\hline Dz8-17 & & & & \\
817_WT & $0.220 \pm 0.015$ & $80.3 \pm 1.2$ & 1.00 & 1.00 \\
817_6 & $0.303 \pm 0.017$ & $79.5 \pm 1.0$ & 1.38 & 0.99 \\
817_10 & $0.313 \pm 0.023$ & $80.0 \pm 1.1$ & 1.42 & 1.00 \\
817_12 & $0.520 \pm 0.036$ & $79.2 \pm 1.1$ & 2.36 & 0.99 \\
817_15 & $0.142 \pm 0.010$ & $81.9 \pm 1.0$ & 0.65 & 1.02 \\
817_150 & $0.070 \pm 0.007$ & $79.3 \pm 2.5$ & 0.32 & 0.99 \\
& & & & \\
Dz10-23 & & & & \\
1023_WT & $0.103 \pm 0.009$ & $86.1 \pm 2.1$ & 1.00 & 1.00 \\
1023_5 & $0.051 \pm 0.008$ & $86.2 \pm 3.8$ & 0.50 & 1.00 \\
1023_9 & $0.050 \pm 0.005$ & $84.6 \pm 2.6$ & 0.49 & 0.98 \\
1023_11 & $0.032 \pm 0.005$ & $87.0 \pm 4.8$ & 0.31 & 1.01 \\
1023_12 & $0.017 \pm 0.004$ & $63.8 \pm 6.8$ & 0.17 & 0.74 \\
1023_15 & $0.033 \pm 0.003$ & $88.3 \pm 3.1$ & 0.32 & 1.03 \\
& & & &
\end{tabular}

DNAzymes (817_6, 817_10, and 817_15) seem to be rather unaffected by the introduction of the modification into the catalytic core - mutants 817_6 and 817_10 display a slight rate improvement of $\sim 40 \%$ as compared to wild-type DNAzyme $8-17$, while in the case of 817_12 a 2-fold improvement can be observed which compares favorably to other modified constructs. On the other hand, 817_15 and 817_150 show an important loss of catalytic activity since the $k_{\text {obs }}$ values dropped by $\sim 40-70 \%$. Interestingly, while the introduction of a 3-deazaadenine residue at $\mathrm{A}_{15.0}$ caused the largest drop in the rate constant, this location had been shown to be rather tolerant to other substitutions such as an abasic site or a C3-spacer. ${ }^{47}$ However, the nonessential $A_{15}$ and $A_{15.0}$ were shown to be responsible for the modulation of the cleavage site selectivity ${ }^{65}$ and to be in close contact with the cleavage site nucleotides (i.e. rG-T, Fig. $1 \mathrm{~A}){ }^{1,66}$ Thus, it seems plausible that deletion of the N3-nitrogens has an impact on the contacts of the catalytic core with the substrate which may account for the drop in activity. In addition, $A_{6}$ located in the AGC loop has been shown to be a critically conserved residue involved in functionally important hydrogen bonds. ${ }^{46,65,67}$ However, $\mathrm{A}_{6}$ appears not to be involved in important interactions via the $\mathrm{N} 3$ atom since the modification only causes a slight variation in $k_{\text {obs }}$.

The inclusion of 3-deazaadenine residues into specific locations of the catalytic core of Dz8-17 has an effect on the catalytic efficiency, which hints at a possible participation of the adenosine N3-nitrogens in the mode of action of Dz8-17 albeit not necessarily in the chemical cleavage step since all $Y_{\mathrm{m}}$ values are unchanged (vide supra). Taken together, substitution at positions $A_{6}, A_{10}$ and $A_{12}$ of Dz8-17 has only a little impact on the catalytic activity while the N3-nitrogens at $A_{15}$ and $A_{15.0}$ seem to be of importance for catalysis. Taking into account that $A_{6}, A_{10}$ and $\mathrm{A}_{12}$ are positioned within the stem-loop motif it seems reasonable that base-pairing is more important and outbalances the loss of N3. On the other hand, $\mathrm{A}_{15}$ and $\mathrm{A}_{15.0}$ are positioned in close proximity to the scissile guanosine (rG) and thus interactions with $2^{\prime}-\mathrm{OH}$ of the substrate might be possible.

For Dz10-23, the maximal cleavage yields of all the modified constructs, except for the mutant 1023_12, reach the same percentage of cleavage as the unmodified DNAzyme $\left(Y_{\mathrm{m}} \sim 85 \%\right)$. The maximal cleavage rate attained only $64 \%$ in the case of the mutant 1023_12. Unlike what has been observed for Dz8-17, the rate constants of all the modified constructs are lower than that of wild-type Dz10-23. For 1023_5 and 1023_9, a rate depletion of around 50\% is observable (Table 3). In the case of 1023_11 and $1023 \_15$ a more marked decrease in the rate constant $(\sim 70 \%)$ is observed, while the catalytic activity is substantially hindered in the case of 1023_12 since a drop of around 80\% is observed.

Previous studies have shown that $\mathrm{A}_{5}$ was a critical residue for maintaining the catalytic activity even though an $A_{5} \rightarrow C_{5}$ mutation was tolerated and it was suggested that one of the nitrogen atoms of the nucleobase was involved in an important hydrogen bond interaction. ${ }^{47-49}$ However, our results $\left(k_{\text {obs }}(\right.$ rel. $)=$ 0.5 for $\mathrm{A}_{5}$ ) and a previous report ${ }^{48}$ indicate that none of the amines located at positions 3 and 6, respectively, play a critical functional role. $A_{9}$ has been found to be rather tolerant to substitution and modification, ${ }^{39,47,68}$ which is reflected by the small $\sim 2$-fold decrease in the rate constant upon substitution with 3-deazaadenine. Moreover, $A_{11}$ was deemed to merely serve as a physical spacer, ${ }^{47}$ but the rather important reduction in $k_{\mathrm{obs}}$ $\left(k_{\text {obs }}(\right.$ rel. $\left.)=0.31\right)$ suggests that $\mathrm{A}_{11}$ might be playing a role in the folding of the DNAzyme into its catalytically active conformation. Deletion of the $\mathrm{N} 3$ atom at $\mathrm{A}_{15}$, located at the $3^{\prime}$-end of the catalytic core in proximity to the scissile bond, causes the catalytic activity to drop to a similar extent as observed for $\mathrm{A}_{11}\left(k_{\mathrm{obs}}(\mathrm{rel}\right.$. $)=$ 0.32 ) even though this base is not thought to be quintessential for catalysis. ${ }^{47}$ Indeed, substitution of $\mathrm{A}_{15}$ with $\mathrm{G}$ or $\mathrm{T}^{48}$ or with a 7-deazaadenine analog equipped with an amino acid side-chain ${ }^{69}$ led to fully active constructs, while the ablation of this nucleobase (abasic site) generated a construct with a very low $k_{\text {obs }}$ but maintained its catalytic activity (i.e. high $Y_{\mathrm{m}}$ value). ${ }^{47}$ Finally, substitution at $\mathrm{A}_{12}$ causes a significant diminution of the rate constant $\left(k_{\text {obs }}(\right.$ rel. $\left.)=0.17\right)$ and 1023_12 is the sole mutant that also showed a diminution of the maximum cleavage yield $\left(Y_{\mathrm{m}}(\mathrm{rel})=\right.$. 0.74 ), hinting at an implication of the N3 atom of this nucleobase in the catalytic mechanism. This is surprising since reports where $\mathrm{A}_{12}$ was substituted with any of the other natural nucleobases ${ }^{48}$ or with 8-aza-7-deazaadenine analogs $\mathrm{s}^{68}$ clearly showed that $\mathrm{A}_{12}$ was probably not crucial for catalytic activity. However, we can suggest that the reduction of the observed rate constant might be consistent with the disruption of one stabilizing hydrogen bond of the transition state since such an event has been estimated to lower the stability by $0.5-2 \mathrm{kcal} \mathrm{mol}^{-1}$ which in turn causes a three- to 25 -fold reduction of the reaction rate. ${ }^{46,70}$ Thus, the N3 atom at $\mathrm{A}_{12}$ of the catalytic core might be involved in critical interactions and might be quite important to catalysis which is reflected by a strong decrease in both $k_{\mathrm{obs}}$ and $Y_{\mathrm{m}}$. Taken together, it appears that the $\mathrm{N} 3$ atoms of $\mathrm{A}_{11}, \mathrm{~A}_{12}$, and $\mathrm{A}_{15}$ all have an effect on the observed rate constants and thus might be involved in the maintenance of the adequate tertiary structure rather than influencing or participating in the chemical cleavage step.

\section{pH-rate profiles}

In order to further investigate the influence of the deletion of the N3 atom on the catalytic efficiency, two modified DNAzymes, 
A)
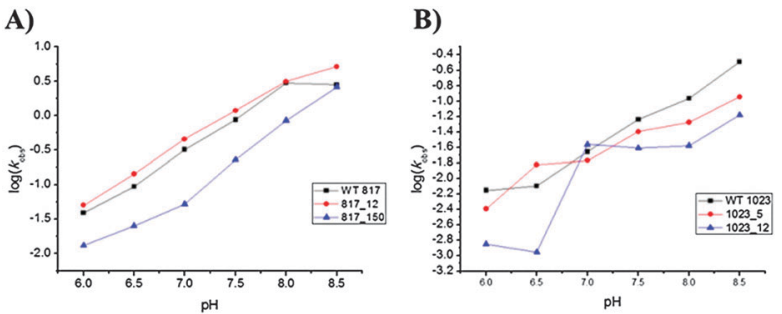

Fig. $3 \mathrm{pH}$ dependence of the observed rate constant for wild-type and mutant DNAzymes. (A) Dz8-17; (B) Dz10-23.

namely 817_12, 817_150, 1023_5 and 1023_12 along with the parent DNAzymes were used for a $\mathrm{pH}$-rate profile analysis. The same kinetic assays as described in the preceding section were carried out at different pHs (namely 6, 6.5, 7, 7.5, 8 and 8.5). The cleavage efficiency was measured as described above and the values for $k_{\mathrm{obs}}$ and $Y_{\mathrm{m}}$ were obtained by curve-fitting. The pH-rate profiles are shown in Fig. 3, Fig. S5 and S6 (ESI $\dagger$ ), while the measured $k_{\mathrm{obs}}$ and $Y_{\mathrm{m}}$ values are summarized in Table S9 (ESI $\dagger$ ). The wild type constructs as well as the modified DNAzymes follow a similar trend since an increase in $k_{\text {obs }}$ with increasing $\mathrm{pH}$ is observed for Dz8-17 as well as for Dz10-23. The plotted values result in the typical linear log $\mathrm{pH}$-rate profiles with a slope of $\sim 1$ for all the investigated Dz8-17 constructs (Fig. 3A and Table S10, ESI $\dagger$ ), and a slight plateau is observed at $\mathrm{pH} \geq 8$ which might arise from a weaker binding of the substrate at higher $\mathrm{pH}$ values as observed for different $8-17$ variants $^{61,71,72}$ and the hammerhead ribozyme. ${ }^{73}$ The preservation of the linear relationship in the $\mathrm{pH}$-rate profiles of all the Dz8-17 constructs suggests that the chemical cleavage step (i.e. transesterification of the embedded labile riboguanosine linkage) rather than a putative conformational change is the rate-determining step as in the case of the parent Dz8-17. ${ }^{54,72-74}$ All the 8-17 DNAzymes that were investigated displayed $k_{\mathrm{obs}}$ values $\left(2.5-5.0 \mathrm{~min}^{-1}\right)$ at $\mathrm{pH} 8.5$ which are considerably higher than the values at $\mathrm{pH}$ 7.0.

Moreover, the $Y_{\mathrm{m}}$ values for wild-type Dz8-17 and 817_12 remained unchanged throughout the entire $\mathrm{pH}$ range, while the mutant 817_150 showed a marked increase in the $Y_{\mathrm{m}}$ value with increasing $\mathrm{pH}$ (from $\sim 50$ to $\sim 80 \%$ ). This observation further confirms that the substitution of the N3-atom by a carbon atom on adenine at position $\mathrm{A}_{12}$ seems to be uncritical and that the N3-atom has no direct effect or involvement in the cleavage process.

Furthermore, this site might be suitable for the inclusion of additional functional groups in order to improve the catalytic efficiency, since higher $k_{\text {obs }}$ values are invariably observed. In contrast, the N3-nitrogen at position $\mathrm{A}_{15.0}$ seems to be of importance for the appropriate catalytic process since there is a clear attenuation of the cleavage efficiency.

A similar trend was observed for Dz10-23 except for 1023_12 where a break in linearity was detected (Fig. 3B). Indeed, $k_{\mathrm{obs}}$ increases steadily with $\mathrm{pH}$ for the wild type as well as for constructs 1023_5 and 1023_12, and the values of the observed rate constants are higher at $\mathrm{pH} 8.5$ than at 7.0, albeit the change is more marked ( $\sim 15$-fold increase) for wild-type than for the modified constructs (2-7-fold increase). This is consistent with the $\mathrm{pH}$-rate profiles reported for wild-type Dz10-23 ${ }^{74,75}$ and modified variants. ${ }^{68}$ Unlike all other modifications, the curve for the construct 1023_12 did not show any linearity but still a clear pH dependency (the pH-rate profiles for Dz1023_12 are the result of five independent experiments). This very distinct curve, compared to the other constructs, might be explained by the protonation state of the nucleobase.

We hypothesize that the protonation state of the nucleobase at position $\mathrm{A}_{12}$ is of importance and plays a crucial role in the enzymatic activity. Indeed, by exchanging the N3-nitrogen with a carbon atom the $\mathrm{p} K_{\mathrm{a}}$ is shifted from $\sim 4.0$ to $\sim 7.0^{76,77}$ which could explain the very low $k_{\text {obs }}$ values observed at $\mathrm{pH} 6$ and 6.5 and the drastic increase at $\mathrm{pH} 7(\sim 20$-fold). In addition, the mutant 1023_12 displays a combination of reduced maximal cleavage yield and reduced $k_{\text {obs }}$ values which might point towards a structural change within the DNAzyme scaffold and therefore might hint at minor groove interactions that arise from the N3-nitrogen at this position. Taken together, it seems that the two investigated constructs of Dz10-23 have a need for the N3 atoms for their catalytic activity and might be involved in transition state stabilization and/or hydrogen bonding with the coordinated inner sphere water molecules of the $\mathrm{Mg}^{2+}$ cation cofactor. ${ }^{54,78,79}$

\section{Conclusions}

The presence of N3-nitrogen atoms of specific residues in the catalytic cores of Dz8-17 and Dz10-23 seems to be of importance for the catalytic activity. The removal of the N3-atoms in some of the conserved adenine nucleobases alters the observed rate constants of both DNAzymes that were investigated. Thus, minor groove interactions seem to play a role in DNAzymes, albeit to a lesser extent than to what has been observed in their RNA counterparts. ${ }^{54}$ Indeed, when the hammerhead ribozyme was subjected to a similar analysis, deletion of the $\mathrm{N} 3$ atom of $\mathrm{A}_{15.0}$ led to a drastic (55-fold) drop in the catalytic activity, ${ }^{54}$ highlighting the importance of hydrogen bonds, hydration and metal bonding to the N3 atoms, which are present in many RNA folds. ${ }^{52}$

When $\mathrm{A}_{15}$ and $\mathrm{A}_{15.0}$ were modified in the catalytic core of Dz8-17, a significant loss in catalytic activity was observed. On the other hand, in Dz10-23 all modifications showed a reduction in the observed rate constant but $\mathrm{A}_{12}$ was found to be the most affected with an $\sim 80 \%$ reduction of activity. Surprisingly, rate enhancement could also be observed for some modifications such as $\mathrm{A}_{12}$ in Dz8-17, thus unraveling a new site for the appendage of functionalities.

The structural features responsible for metal-specificity and binding in DNAzymes have still not been fully identified. ${ }^{80}$ On the other hand, N7 and N9 sites have been shown to be important metal binding sites in various nucleic acids, especially in ribozymes. ${ }^{81,82}$ In addition, adenine analogs have been used to highlight the coordination potential of more unusual binding sites such as the N3 nitrogens of adenine. ${ }^{83,84}$ Whilst our data do not suggest a direct binding of the $\mathrm{Mg}^{2+}$ cations to N3-nitrogens of adenine, these atoms could be involved in 
coordination to the 2 '-OH units of the scissile residues of the substrates (especially $\mathrm{A}_{15.0}$ in Dz8-17), to water molecules coordinated in the inner sphere of the $\mathrm{Mg}^{2+}$ cofactor, or to water molecules that are part of the ribbon of hydration. ${ }^{85}$ The identification of critical positions - together with previously identified important nucleotides within the catalytic cores of Dz8-17 and Dz10-23 - reinforces the assumption that the network of hydrogen bonding and other interactions is a fragile structure, influenced by many parameters and it plays an important role in the catalytic mechanism of nucleic acid enzymes. The generality of these observations still needs to be extended to other catalytic DNAs (e.g. bipartite ${ }^{86}$ or $614^{87}$ DNAzymes) and studies towards this aim as well as more detailed kinetic investigations (e.g. to assess the effect of metal cofactors, role of the $\mathrm{O} 2$ functionalities of $\mathrm{dT}$ residues ${ }^{88}$ etc.) are currently underway. Nonetheless, this study reveals that these 'A-minor like' interactions might be regarded as another protagonist within the catalytic mechanism of DNAzymes.

\section{Experimental}

\section{General procedures}

All chemicals and solvents used were purchased from Sigma, AK Scientific Inc. or Alfa Aesar, unless stated otherwise. All reactions were performed under Ar in flame-dried glassware. Anhydrous solvents for reactions were obtained by filtration through activated aluminum oxide, or by storage over $4 \AA$ activated molecular sieves. Flash chromatography was performed using SiliaFlash ${ }^{\circledR}$ P60 (230-400 mesh) from Silicycle. Thin layer chromatography was carried out on precoated glass-backed plates of silica gel $\left(0.25 \mathrm{~mm}, \mathrm{UV}_{254}\right)$ from Macherey-Nagel. NMR spectra were recorded at RT on a Bruker DRX-400 or a Bruker AC-300 spectrometer (400 or $300 \mathrm{MHz}$ for ${ }^{1} \mathrm{H}$, 101 or $75.5 \mathrm{MHz}$ for ${ }^{13} \mathrm{C}$, 121.4 MHz for ${ }^{31} \mathrm{P}$, and $376.5 \mathrm{MHz}$ for ${ }^{19} \mathrm{~F}$ ) and all spectra were referenced to the signals of the corresponding solvent. Chemical shifts are given in ppm ( $\delta$ scale) and coupling constants $(J)$ in $\mathrm{Hz}$. Multiplicities are abbreviated as follows: $\mathrm{s}=$ singlet, $\mathrm{d}=$ doublet, $\mathrm{t}=$ triplet, $\mathrm{q}=$ quadruplet, $\mathrm{m}=$ multiplet, and $\mathrm{br}=$ broad. High resolution electrospray ionization (ESI) mass spectra (MS, $m / z$ ) were recorded on a Thermo Scientific LTQ Orbitrap XL instrument. MALDI-TOF spectra were recorded on an Applied Biosystems Sciex QSTAR Pulsar instrument. Natural nucleoside phosphoramidites were purchased from Tides Service Technology and the solid supports were from Glen Research.

For the determination of the concentration of the oligonucleotides a Perkin Elmer Lambda 16 UV/Vis spectrometer was used. The concentrations were obtained by using the absorbance at $260 \mathrm{~nm}\left(A_{260}\right)$. Thermal denaturation experiments were carried out on a Varian Cary 3E UV/Vis spectrometer. Absorbances were monitored at $260 \mathrm{~nm}$. The heating/cooling rate was set to $0.5{ }^{\circ} \mathrm{C}$ per minute and all samples were covered with mineral oil to prevent evaporation of the solution during the heating process. The program was set to a heating-cooling-cycle starting at $25{ }^{\circ} \mathrm{C}$ and heating to $90{ }^{\circ} \mathrm{C}$. The first derivatives of the melting curves were calculated using the Variant software. The phosphorimager plates were analyzed on a Storm 820 Phosphorimager from Amersham Molecular Devices using the ImageQuant software (both from GE Healthcare). Unmodified oligonucleotides were purchased from Microsynth and gel purified (PAGE 20\%). Acrylamide/bisacrylamide (19:1, 40\%) was obtained from Serva. T4 Polynucleotide kinase was purchased from Promega and $\gamma^{32}$ P-ATP from Hartmann Analytics.

\section{Synthesis of phosphosphoramidite}

The 3-deazaadenine nucleobase was synthesized following a literature procedure. ${ }^{57}$ The preparation of 3 -deazaadenine phosphoramidite was carried out by variation of a literature procedure. ${ }^{56}$ Briefly, to a flask containing the DMTr-protected precursor (100 mg, $0.15 \mathrm{mmol}, 1$ eq.) dissolved in ACN (3 mL), $N$-ethyldiisopropylamine $(0.075 \mathrm{~mL}, 0.55 \mathrm{mmol}, 3 \mathrm{eq}$.) was slowly added, followed by a dropwise addition of 2-cyanoethyl- $N, N$ diisopropylchlorophosphoramidite ( $0.048 \mathrm{~mL}, 0.28 \mathrm{mmol}, 1.5 \mathrm{eq}$.). The reaction was stirred at RT until TLC showed a complete conversion of the precursor. The reaction was then extracted with $\mathrm{NaHCO}_{3}$, filtered, and dried over $\mathrm{MgSO}_{4}$. Volatiles were evaporated in vacuo. The white solid was purified by flash column chromatography eluting with EtOAc: hexanes 6:4 +1\% TEA to afford the 3-deazaadenine phosphoramidite in $50 \%$ yield ( $65 \mathrm{mg}$ ). Data for 3-deazaadenine phosphoramidite:

${ }^{1} \mathrm{H}-\mathrm{NMR}\left(300 \mathrm{MHz}, \mathrm{CDCl}_{3}\right) \delta 8.14(\mathrm{dd}, J=5.6,4.2 \mathrm{~Hz}, 1 \mathrm{H})$, $8.10(\mathrm{~s}, 1 \mathrm{H}), 7.63(\mathrm{~d}, J=5.6 \mathrm{~Hz}, 1 \mathrm{H}), 7.40(\mathrm{~d}, J=7.5 \mathrm{~Hz}, 2 \mathrm{H}), 7.27$ (ddd, $J=12.2,5.7,2.6 \mathrm{~Hz}, 9 \mathrm{H}), 6.83-6.77(\mathrm{~m}, 4 \mathrm{H}), 6.31-6.23$ (m, $1 \mathrm{H}), 4.80-4.72(\mathrm{~m}, 1 \mathrm{H}), 4.32-4.24(\mathrm{~m}, 1 \mathrm{H}), 3.77$ (d, $J=1.3 \mathrm{~Hz}$, $7 \mathrm{H}), 3.72-3.53(\mathrm{~m}, 4 \mathrm{H}), 3.45-3.36(\mathrm{~m}, 2 \mathrm{H}), 2.67-2.59(\mathrm{~m}, 3 \mathrm{H})$, $2.46(\mathrm{t}, J=6.3 \mathrm{~Hz}, 1 \mathrm{H}), 1.37(\mathrm{~s}, 12 \mathrm{H}), 1.21-1.16(\mathrm{~m}, 10 \mathrm{H}), 1.11$ (d, $J=6.8 \mathrm{~Hz}, 3 \mathrm{H}) .{ }^{31} \mathrm{P}-\mathrm{NMR}\left(122 \mathrm{MHz}, \mathrm{CDCl}_{3}\right) \delta=149.29$, 149.27 ppm. HR-MS $\left(\right.$ ESI $\left.^{+}\right) \mathrm{m} / z$ : calc. for $\mathrm{C}_{49} \mathrm{H}_{59} \mathrm{~N}_{6} \mathrm{O}_{8} \mathrm{P}(\mathrm{M}+\mathrm{H})^{+}$: 891.4205; found: 891.4195 .

\section{Oligonucleotide synthesis}

All oligonucleotides were synthesized by standard solid-phase phosphoramidite chemistry on a $1.3 \mu \mathrm{mol}$ scale using a Pharmacia LKB Gene Assembler Special DNA Synthesizer with 5-(ethylthio)$1 H$-tetrazole $(0.25 \mathrm{M}$ in $\mathrm{ACN})$ as the activator and a coupling time of $90 \mathrm{~s}$ for the natural phosphoramidites and $12 \mathrm{~min}$ for the 3-deazaadenine phosphoramidite. The oligomers were deprotected using a $33 \% \mathrm{NH}_{3}$ solution for $0.5 \mathrm{~h}$ at room temperature followed by $16 \mathrm{~h}$ at $55{ }^{\circ} \mathrm{C}$ in a screw capped $1.5 \mathrm{~mL}$ tube. The oligonucleotides were filtered through $13 \mathrm{~mm}$ Yeti HPLC filters (PTFE membrane, pore size $0.45 \mu \mathrm{m}$ ). Subsequently, the samples were precipitated with ethanol and purified by $15 \%$ polyacrylamide gel electrophoresis. Desalting was achieved using Sephadex G-10 spin columns.

\section{Kinetic assays}

The cleavage reactions were performed under single turnover conditions. The reactions were carried out in $10 \mu \mathrm{L}$ of HEPES buffer $10 \times\left(0.5 \mathrm{M}\right.$ HEPES, $2 \mathrm{M} \mathrm{NaCl}, 100 \mathrm{mM} \mathrm{MgCl}_{2}$, pH 7.2 for Dz8.17 and 0.5 M HEPES, $2 \mathrm{M} \mathrm{NaCl}, 500 \mathrm{mM} \mathrm{MgCl}_{2}$, pH 8 for Dz10-23) at room temperature. Rather high $\left[\mathrm{Mg}^{2+}\right]$ were chosen for Dz10-23, so that the rate constants are $>10^{-2} \mathrm{~min}^{-1} .^{47}$ 
Indeed, when Dz10-23 is assayed at low $\left[\mathrm{Mg}^{2+}\right]$ and under single-turnover conditions with DNA-RNA chimeric substrates, $k_{\text {obs }}$ are usually in the $10^{-2}$ to $10^{-3} \mathrm{~min}^{-1}$ range. ${ }^{38,39}$ A mixture of $60 \mathrm{pmol}$ of DNAzyme, $10 \mu \mathrm{L}$ of buffer and $\mathrm{H}_{2} \mathrm{O}$ was prepared in an Eppendorf tube and pre-heated at $37{ }^{\circ} \mathrm{C} .2 \mu \mathrm{L}$ of the ${ }^{32}$ P-radiolabeled substrate was mixed with 0.5 pmol of the unlabeled substrate and incubated at $37{ }^{\circ} \mathrm{C}$ prior to the reaction. $2.5 \mu \mathrm{L}$ of the substrate mixture was added to the reaction mixture to initiate the reactions in a total reaction volume of $100 \mu \mathrm{L}$. At each time point, $5 \mu \mathrm{L}$ of the reaction mixtures was either quenched in an equal volume of stop solution (70\% formamide, 30\% EDTA $0.5 \mathrm{M}$, pH 8.0, 0.1\% bromophenol, $0.1 \%$ xylene cyanol) for Dz8-17 or in $5 \mu \mathrm{L}$ of EDTA $0.5 \mathrm{M}, \mathrm{pH}$, followed by the addition of NaOAc ( $300 \mathrm{mM}$ final concentration) and $500 \mu \mathrm{L}$ of EtOH and precipitation by centrifugation for Dz10-23. For Dz8-17, Dz10-23 and all modified versions timepoints were taken after $7,15,30 \mathrm{~s}, 1,2,5,10,20,30,60$, and $120 \mathrm{~min}$. At least three individual experiments were conducted for each construct and the averaged results are taken. The reactions were analyzed by $20 \%$ polyacrylamide gel electrophoresis. Visualization was carried out by means of a phosphorimager and polygons were drawn around the bands corresponding to the cleaved and uncleaved species using the ImageQuant software. The obtained data were then fitted to first-order reactions using the OriginPro (version 8.0) software using the equation $Y=Y_{\mathrm{m}}\left(1-\mathrm{e}^{-k t}\right)$, where $k\left(k_{\mathrm{obs}}\right)$ is the observed rate constant (in $\min ^{-1}$ ), $Y$ the cleavage yield (in \%) at any given time-point $t$, and $Y_{\mathrm{m}}$ the maximum cleavage yield. All errors are given as the averaged standard deviation from the exponential fit of each time-course.

\section{Acknowledgements}

This work was supported by a grant from the Swiss National Science Foundation (grant-no. PZ00P2_144595 to M.H.). We thank Prof. C. Leumann for providing the lab space and equipment, as well as for his constant support, fruitful discussions and critical assessment of this manuscript.

\section{Notes and references}

1 K. Schlosser and Y. Li, Chem. Biol., 2009, 16, 311-322.

2 S. K. Silverman, Angew. Chem., Int. Ed., 2010, 49, 7180-7201.

3 X.-B. Zhang, R.-M. Kong and Y. Lu, Annu. Rev. Anal. Chem., 2011, 4, 105-128.

4 W. L. Ward, K. Plakos and V. J. DeRose, Chem. Rev., 2014, 114, 4318-4342.

5 D. Balke, C. Wichert, B. Appel and S. Müller, Appl. Microbiol. Biotechnol., 2014, 98, 3389-3399.

6 R. R. Breaker and G. F. Joyce, Chem. Biol., 1994, 1, 223-229.

7 S. W. Santoro and G. F. Joyce, Proc. Natl. Acad. Sci. U. S. A., 1997, 94, 4262-4266.

8 K. Schlosser and Y. Li, ChemBioChem, 2010, 11, 866-879.

9 M. Chandra, A. Sachdeva and S. K. Silverman, Nat. Chem. Biol., 2009, 5, 718-720.
10 H. Gu, K. Furukawa, Z. Weinberg, D. F. Berenson and R. R. Breaker, J. Am. Chem. Soc., 2013, 135, 9121-9129.

11 B. M. Brandsen, A. R. Hesser, M. A. Castner, M. Chandra and S. K. Silverman, J. Am. Chem. Soc., 2013, 135, 16014-16017.

12 J. Chandrasekar and S. K. Silverman, Proc. Natl. Acad. Sci. U. S. A., 2013, 110, 5315-5320.

13 M. Chandra and S. K. Silverman, J. Am. Chem. Soc., 2008, 130, 2936-2937.

14 P. I. Pradeepkumar, C. Höbartner, D. A. Baum and S. K. Silverman, Angew. Chem., Int. Ed., 2008, 47, 1753-1757.

15 R. L. Coppins and S. K. Silverman, Nat. Struct. Mol. Biol., 2004, 11, 270-274.

16 W. R. Purtha, R. L. Coppins, M. K. Smalley and S. K. Silverman, J. Am. Chem. Soc., 2005, 127, 13124-13125.

17 Y. F. Li and R. R. Breaker, Proc. Natl. Acad. Sci. U. S. A., 1999, 96, 2746-2751.

18 L. Büttner, F. Javadi-Zarnaghi and C. Höbartner, J. Am. Chem. Soc., 2014, 136, 8131-8137.

19 D. J. Chinnapen and D. Sen, Proc. Natl. Acad. Sci. U. S. A., 2004, 101, 65-69.

20 V. B. Pinheiro and P. Holliger, Curr. Opin. Chem. Biol., 2012, 16, 245-252.

21 V. B. Pinheiro and P. Holliger, Trends Biotechnol., 2014, 32, 321-328.

22 M. Hollenstein, Molecules, 2012, 17, 13569-13591.

23 S. W. Santoro, G. F. Joyce, K. Sakthivel, S. Gramatikova and C. F. Barbas, J. Am. Chem. Soc., 2000, 122, 2433-2439.

24 D. M. Perrin, T. Garestier and C. Hélène, J. Am. Chem. Soc., 2001, 123, 1556-1563.

25 A. V. Sidorov, J. A. Grasby and D. M. Williams, Nucleic Acids Res., 2004, 32, 1591-1601.

26 M. Hollenstein, C. Hipolito, C. Lam, D. Dietrich and D. M. Perrin, Angew. Chem., Int. Ed., 2008, 47, 4346-4350.

27 M. Hollenstein, C. J. Hipolito, C. H. Lam and D. M. Perrin, Nucleic Acids Res., 2009, 37, 1638-1649.

28 M. Hollenstein, C. J. Hipolito, C. H. Lam and D. M. Perrin, ChemBioChem, 2009, 10, 1988-1992.

29 C. H. Lam, C. J. Hipolito, M. Hollenstein and D. M. Perrin, Org. Biomol. Chem., 2011, 9, 6949-6954.

30 C. J. Hipolito, M. Hollenstein, C. H. Lam and D. M. Perrin, Org. Biomol. Chem., 2011, 9, 2266-2273.

31 M. Hollenstein, C. J. Hipolito, C. H. Lam and D. M. Perrin, ACS Comb. Sci., 2013, 15, 174-182.

32 A. I. Taylor, V. B. Pinheiro, M. J. Smola, A. S. Morgunov, S. Peak-Chew, C. Cozens, K. M. Weeks, P. Herdewijn and P. Holliger, Nature, 2015, 518, 427-430.

33 M. Sioud and M. Leirdal, J. Mol. Biol., 2000, 296, 937-947.

34 B. Vester, L. B. Lundberg, M. D. Sørensen, B. R. Babu, S. Douthwaite and J. Wengel, J. Am. Chem. Soc., 2002, 124, 13682-13683.

35 S. Schubert, D. C. Gül, H.-P. Grunert, H. Zeichhardt, V. A. Erdmann and J. Kurreck, Nucleic Acids Res., 2003, 31, 5982-5992.

36 C. H. Lam and D. M. Perrin, Bioorg. Med. Chem. Lett., 2010, 20, 5119-5122. 
37 L. Robaldo, F. Izzo, M. Dellafiore, C. Proietti, P. V. Elizalde, J. M. Montserrat and A. M. Iribarren, Bioorg. Med. Chem., 2012, 20, 2581-2586.

38 Y. Liu, Z. Li, G. Liu, Q. Wang, W. Chen, D. Zhang, M. Cheng, Z. Zheng, K. Liu and J. He, Chem. Commun., 2013, 49, 5037-5039.

39 Z. Li, Y. Liu, G. Liu, J. Zhu, Z. Zheng, Y. Zhou and J. He, Bioorg. Med. Chem., 2014, 22, 4010-4017.

40 V. Gheerardijn, J. Van den Begin and A. Madder, Beilstein J. Org. Chem., 2014, 10, 2566-2572.

41 L. Robaldo, A. Berzal-Herranz, J. M. Montserrat and A. M. Iribarren, ChemMedChem, 2014, 9, 2172-2177.

42 D. B. Kearns, Trends Microbiol., 2013, 21, 508-509.

43 H.-K. Kim, J. Liu, J. Li, N. Nagraj, M. Li, C. M.-B. Pavot and Y. Lu, J. Am. Chem. Soc., 2007, 129, 6896-6902.

44 H.-K. Kim, I. Rasnik, J. Liu, T. Ha and Y. Lu, Nat. Chem. Biol., 2007, 3, 763-768.

45 J. C. F. Lam and Y. Li, ChemBioChem, 2010, 11, 1710-1719.

46 A. Peracchi, M. Bonaccio and M. Clerici, J. Mol. Biol., 2005, 352, 783-794.

47 B. Wang, L. Q. Cao, W. Chiuman, Y. F. Li and Z. Xi, Biochemistry, 2010, 49, 7553-7562.

48 Z. Zaborowska, J. P. Fürste, V. A. Erdmann and J. Kurreck, J. Biol. Chem., 2002, 43, 40617-40622.

49 Z. Zaborowska, S. Schubert, J. Kurreck and V. A. Erdmann, FEBS Lett., 2005, 579, 554-558.

50 H. W. Pley, K. M. Flaherty and D. B. McKay, Nature, 1994, 111-113.

51 P. Nissen, J. A. Ippolito, N. Ban, P. B. Moore and T. A. Steitz, Proc. Natl. Acad. Sci. U. S. A., 2001, 98, 4899-4903.

52 J. K. Soukup, N. Minakawa, A. Matsuda and S. A. Strobel, Biochemistry, 2002, 41, 10426-10438.

53 S. E. Butcher and A. M. Pyle, Acc. Chem. Res., 2011, 44, 1302-1311.

54 S. Bevers, G. Xiang and L. W. McLaughlin, Biochemistry, 1996, 35, 6483-6490.

55 C. Lever, X. Li, R. Cosstick, S. Ebel and T. Brown, Nucleic Acids Res., 1993, 21, 1743-1746.

56 K. J. Salandria, J. W. Arico, A. K. Calhoun and L. W. McLaughlin, J. Am. Chem. Soc., 2011, 133, 1766-1768.

57 C. K. Crey-Desbiolles and M. Kotera, Bioorg. Med. Chem., 2006, 14, 1935-1941.

58 S. K. Silverman, Nucleic Acids Res., 2005, 33, 6151-6163.

59 J. C. F. Lam, S. O. Kwan and Y. Li, Mol. BioSyst., 2011, 7, 2139-2146.

60 D. Mazumdar, N. Nagraj, H.-K. Kim, X. Meng, A. K. Brown, Q. Sun, W. Li and Y. Lu, J. Am. Chem. Soc., 2009, 131, 5506-5515.

61 M. Bonaccio, A. Credali and A. Peracchi, Nucleic Acids Res., 2004, 32, 916-925.
62 I. Hirao, Y. Nishimura, Y. Tagawa, K. Watanabe and K. Miura, Nucleic Acids Res., 1992, 20, 3891-3896.

63 K. E. Nelson, P. J. Bruesehoff and Y. Lu, J. Mol. Evol., 2005, 61, 216-225.

64 J. Nowakowski, P. J. Shim, G. S. Prasad, C. D. Stout and G. F. Joyce, Nat. Struct. Biol., 1999, 6, 151-156.

65 K. Schlosser, J. Gu, L. Sule and Y. Li, Nucleic Acids Res., 2008, 36, 1472-1481.

66 Y. Liu and D. Sen, J. Mol. Biol., 2008, 381, 845-859.

67 R. P. G. Cruz, J. B. Withers and Y. Li, Chem. Biol., 2004, 11, 57-67.

68 J. He, D. Zhang, Q. Wang, X. Wei, M. Cheng and K. Liu, Org. Biomol. Chem., 2011, 9, 5728-5736.

69 D. Smuga, K. Majchrzak, E. Sochacka and B. Nawrot, New J. Chem., 2010, 34, 934-948.

70 A. R. Fersht and L. Serrano, Curr. Opin. Struct. Biol., 1993, 3, 75-83.

71 A. K. Brown, J. Li, C. M.-B. Pavot and Y. Lu, Biochemistry, 2003, 42, 7152-7161.

72 K. Schlosser and Y. Li, Nucleic Acids Res., 2009, 37, 413-420.

73 S. C. Dahm, W. B. Derrick and O. C. Uhlenbeck, Biochemistry, 1993, 32, 13040-13045.

74 N. Ota, M. Warashina, K. Hirano, K. Hatanaka and K. Taira, Nucleic Acids Res., 1998, 26, 3385-3391.

75 S. W. Santoro and G. F. Joyce, Biochemistry, 1998, 37, 13330-13342.

76 M. Ikehara, T. Fukui and S. Uesugi, J. Biochem., 1974, 76, 107-115.

77 Y. H. Jang, K. N. Rogstad, L. C. Sowers and W. A. Goddard, Chem. Res. Toxicol., 2003, 16, 1455-1462.

78 S. Kolev, P. S. Petkov, M. Rangelov and G. N. Vayssilov, ACS Chem. Biol., 2013, 8, 1576-1589.

79 W. T. D. Lin, P.-J. J. Huang, R. Pautler and J. Liu, Chem. Commun., 2014, 50, 11859-11862.

80 M. Cepeda-Plaza, E. L. Null and Y. Lu, Nucleic Acids Res., 2013, 41, 9361-9370.

81 B. Lippert, Coord. Chem. Rev., 2000, 200-202, 487-516.

82 M. Warashina, T. Kuwabara, Y. Nakamatsu, Y. Takagi, Y. Kato and K. Taira, J. Am. Chem. Soc., 2004, 126, 12291-12297.

83 A. Houlton, Adv. Inorg. Chem., 2002, 53, 87-158.

84 M. A. Galindo, D. Amantia, A. M. Martinez, W. Clegg, R. W. Harrington, V. M. Martinez and A. Houlton, Inorg. Chem., 2009, 48, 10295-10303 and references therein.

85 V. Tereshko, G. Minasov and M. Egli, J. Am. Chem. Soc., 1999, 121, 3590-3595.

86 A. R. Feldman and D. Sen, J. Mol. Biol., 2001, 313, 283-294. 87 M. A. Carrigan, A. Ricardo, D. N. Ang and S. A. Benner, Biochemistry, 2004, 43, 11446-11459.

88 T. Lan and L. W. McLaughlin, Biochemistry, 2001, 40, 968-976. 